\title{
ON THE COMPLETION OF UNIFORM CONVERGENCE SPACES AND AN APPLICATION TO NONLINEAR PDES
}

\author{
J H VAN DER WALT
}

\begin{abstract}
We obtain a characterization of the completion of an initial uniform convergence structure, which includes, among others, subspaces and projective limits of uniform convergence spaces. In this regard, there seems to be a gap in the literature. This characterization is obtained in a surprisingly straightforward way, and this should be viewed as a consequence of the fact that the category UCS of all uniform convergence spaces is Cartesian closed [6], as apposed to the category UTS consisting of all uniform topological spaces. As an application, we give an existence and regularity result for the solutions of nonlinear PDEs.
\end{abstract}

\section{INTRODUCTION}

Uniform spaces, and more generally uniform convergence spaces, appear in many important applications of topology, and in particular analysis. In this regard, the concepts of completeness and completion of a uniform convergence space play a central role. Indeed, Baire's celebrated Category Theorem asserts that every complete metric space cannot be expressed as the union of a countable family of closed nowhere dense sets. The importance of this result is demonstrated by the fact that the Banach-Steinhauss Theorem, as well as the Closed Graph Theorem in Banach spaces follow from it.

However, in many situations one deals with a space $X$ which is incomplete, and in these cases one may want to construct the completion of $X$. In this regard, the main result, see for instance [6], is that every Hausdorff uniform convergence space $X$ may be uniformly continuously embedded into a complete, Hausdorff uniform convergence space $X^{\sharp}$ in such a way that the image of $X$ in $X^{\sharp}$ is dense. Moreover, for every complete uniform convergence space $Y$, and any uniformly continuous mapping

$$
F: X \rightarrow Y
$$

the diagram

Key words and phrases. Uniform convergence spaces, completions, nonlinear PDEs. 


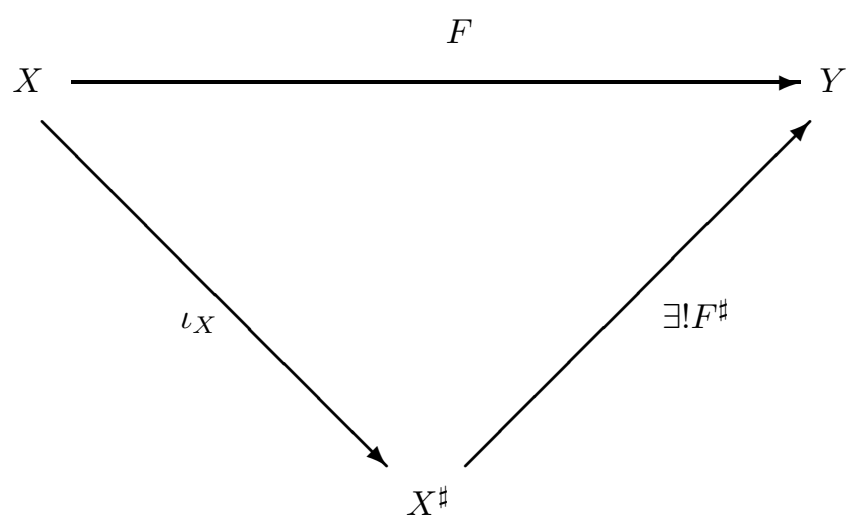

commutes, with $F^{\sharp}$ uniformly continuous, and $\iota_{X}$ the canonical embedding of $X$ into its completion $X^{\sharp}$.

It is often not only the completion $X^{\sharp}$ of a uniform convergence space $X$ that is of interest, but also the extension $F^{\sharp}$ of a uniformly continuous mapping from $X$ to $X^{\sharp}$. In this regard, we recall that one of the major applications of uniform spaces, and recently, see [14 and [15], also uniform convergence spaces, is to the solutions of PDEs. Indeed, let us consider a PDE

$$
T u=f
$$

with $T$ the possibly nonlinear PDE operator which acts on some relatively small space $X$ of classical functions, $u$ the unknown function, while the right hand term $f$ belongs to some space $Y$. One usually considers some uniformities, or more generally uniform convergence structures, on $X$ and $Y$ in such a way that the mapping

$$
T: X \rightarrow Y
$$

is uniformly continuous. It is well known that the equation (11) can have solutions of physical interest which, however, may fail to be classical, in the sense that they do not belong to $X$. From here, therefore, the particular interest in generalized solutions to (11). Such generalized solutions to (11) may be obtained by constructing the completions $X^{\sharp}$ and $Y^{\sharp}$ of $X$ and $Y$, respectively. The mapping (2) extends uniquely to a mapping

$$
T^{\sharp}: X^{\sharp} \rightarrow Y^{\sharp}
$$

so that the diagram 


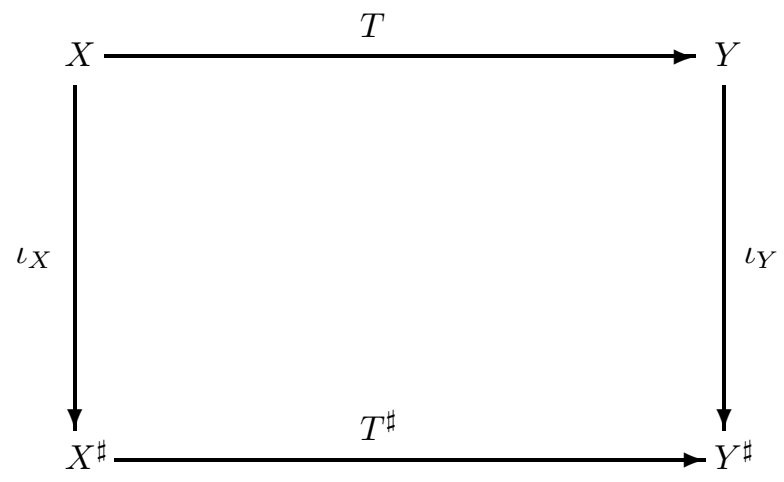

commutes. One may now consider the extended equation

$$
T^{\sharp} u^{\sharp}=f
$$

where the generalized solutions to (10) are the solutions to (4). Note that the existence of generalized solutions depends on the properties of the mapping $T^{\sharp}$, as opposed to the regularity of the generalized solutions, which may be interpreted as the extent to which a generalized solution exhibits characteristics of classical solutions, which depends on the properties of the space $X^{\sharp}$ and its elements. It is therefore clear that not only the completion $X^{\sharp}$ of a uniform convergence space $X$, but also the the associated extensions of uniformly continuous mappings, defined on $X$, are of interest.

The example given above indicates a particular point of interest. The uniform convergence structure $\mathcal{J}_{X}$ on the domain $X$ of the PDE operator $T$ is usually defined as the initial uniform convergence structure 4 ] with respect to some uniform convergence structure $\mathcal{J}_{Y}$ on $Y$, and a family of mappings

$$
\left(\psi_{i}: X \rightarrow Y\right)_{i \in I}
$$

In the case of PDEs, the mappings $\psi_{i}$ are usually some partial differential operators. A natural question arises as to the connection between the completion of $X$, and the completion of $Y$. More generally, consider a set $X$, a family of mappings

$$
\left(\psi_{i}: X \rightarrow X_{i}\right)_{i \in I}
$$

where each $X_{i}$ is a uniform convergence space. If the family $\left(\psi_{i}\right)_{i \in I}$ separates the points of $X$, then the initial uniform convergence structure on $X$ with respect to this family of mappings is also Hausdorff, and we may consider its completion $X^{\sharp}$. In this regard there seems to be a gap in the literature. We aim to clarify the connection between the completion $X^{\sharp}$ of $X$, and the completions $X_{i}^{\sharp}$ of the $X_{i}$. We note the relative ease with which we obtain the characterization, which is essentially due to the fact that the category UCS is Cartesian closed.

Regarding the above example concerning possibly nonlinear PDEs, we note that the uniform structure on the target space $Y$ is usually induced by some locally convex linear space topology on $Y$, while the initial uniform structure on $X$ is defined in terms of some linear partial differential operator. Indeed, the Sobolev space $H^{1}(\Omega)$, for instance, may be defined as the completion of the initial uniform 
structure on $\mathcal{C}^{1}(\Omega)$ with respect to the family of mappings

$$
\left(D^{\alpha}: \mathcal{C}^{1} \rightarrow \mathcal{L}_{2}(\Omega)\right)_{|\alpha| \leq 1}
$$

These methods, however, fail to deliver the existence of generalized solutions to any significantly general class of PDEs, particularly in the nonlinear case. This is not due to any conceptual obstacles, and even less so to the limitations of MATHEMATICS as such, but rather to the inherent limitations of the linear function analytic methods themselves.

Indeed, the general, type independent theory [11 for the existence of solutions to nonlinear PDEs, delivers generalized solutions to very large classes of equations as elements of the Dedekind completion of suitably constructed partially ordered sets. What is more, one obtains a blanket regularity for these generalized solutions, as they may be assimilated with Hausdorff continuous, interval valued functions [1]. As an application of the results on the completion of uniform convergence spaces, we present a significant enrichment of the basic theory of Order Completion [11. In this regard, we obtain the existence and uniqueness of generalized solutions to $\mathcal{C}^{\infty}$-smooth, nonlinear PDEs, which may be assimilated with functions which are $\mathcal{C}^{\infty}$-smooth everywhere except on some closed nowhere dense set.

The paper is organized as follows. Section 2 deals with preliminary results on the extensions of uniformly continuous mappings. In Section 3 we characterize the completion of the initial uniform convergence structure on a set $X$, with respect to uniform convergence structures $\mathcal{J}_{i}$ on sets $X_{i}$, and a family of mappings

$$
\left(\psi_{i}: X \rightarrow X_{i}\right)_{i \in I}
$$

In the context of nonlinear PDEs, as explained above, this characterization may be viewed as a regularity result. In Section 4 we apply the results of Sections 2 and 3 to nonlinear PDEs.

\section{Extensions of Uniformly Continuous Mappings}

In this section we obtain a preliminary result concerning the extension

$$
F^{\sharp}: X^{\sharp} \rightarrow Y^{\sharp}
$$

of a uniformly continuous mapping

$$
F: X \rightarrow Y
$$

from a uniform convergence space $X$ into a uniform convergence space $Y$. All uniform convergence spaces are assumed Hausdorff. The main application of the results we obtain in this section will be to the realization of the completion of certain classes of uniform convergence spaces, which is the subject of the next section. First we note that the completion $X^{\sharp}$ of a subspace $X$ of a uniform convergence space $Y$ is uniformly isomorphic to a subspace of the completion $Y^{\sharp}$ of $Y$. In the case of a uniform space this is obvious. Indeed, $X$ is uniformly homeomorphic to a subspace $\iota_{Y}(X)$ of $Y^{\sharp}$. Moreover, $X$ is dense in the closure of $\iota(X)$, which is complete. For uniform convergence spaces in general, this is not quite as obvious. Recall that a subspace $X$ of a convergence space $Y$ need not be dense in its closure, where the closure of $X$ is the smallest closed subspace of $Y$ which contains $X$. Never the less, the proof of the following proposition is particularly simple. 
Proposition 2.1. Let $X$ be a subspace of the uniform convergence space $Y$. Then there is a subspace $X^{\sharp}$ of the completion $Y^{\sharp}$ of $Y$ such that $X^{\sharp}$ is the completion of $X$.

Proof. Let $\iota_{Y}: Y \rightarrow Y^{\sharp}$ be the uniformly continuous embedding associated with the completion $Y^{\sharp}$ of $Y$, and $\iota_{X}: X \rightarrow X^{\sharp}$ the uniformly continuous embedding associated with the completion $X^{\sharp}$ of $X$. Let $i: X \rightarrow Y$ denote the inclusion mapping, which is of course a uniformly continuous embedding. Then the diagram

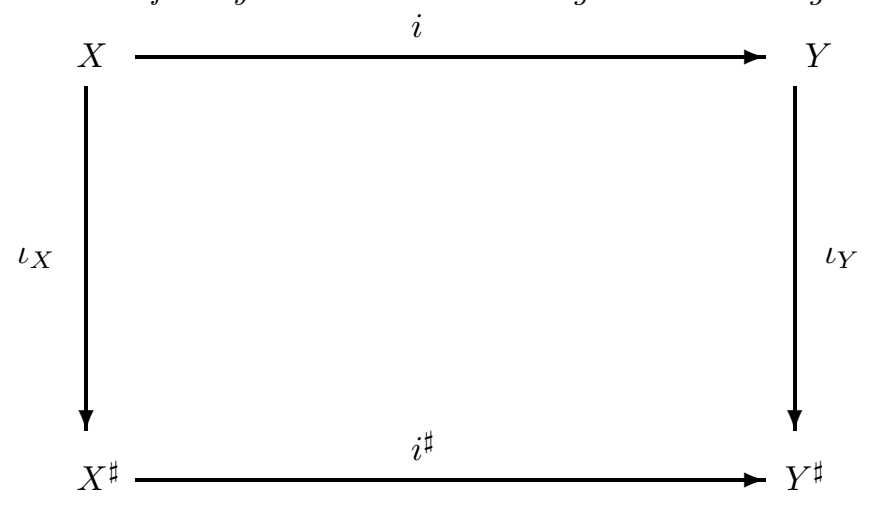

commutes, with $i^{\sharp}$ uniformly continuous. We claim that $i^{\sharp}$ is a uniformly continuous embedding. First we show that $i^{\sharp}$ is injective. To this end, consider any $x_{0}^{\sharp}, x_{1}^{\sharp} \in X^{\sharp}$ and suppose that

$$
i^{\sharp}\left(x_{0}^{\sharp}\right)=i^{\sharp}\left(x_{1}^{\sharp}\right)=y^{\sharp}
$$

for some $y^{\sharp} \in Y^{\sharp}$. Since $\iota_{X}(X)$ is dense in $X^{\sharp}$ there exists Cauchy filters $\mathcal{F}$ and $\mathcal{G}$ on $X$ such that $\iota_{X}(\mathcal{F})$ converges to $x_{0}^{\sharp}$ and $\iota_{X}(\mathcal{G})$ converges to $x_{1}^{\sharp}$. From the diagram above it follows that $\iota_{Y}(i(\mathcal{F}))$ and $\iota_{Y}(i(\mathcal{G}))$ converges to $y^{\sharp}$. Therefore the filter

$$
\mathcal{H}=\iota_{Y}(i(\mathcal{F})) \cap \iota_{Y}(i(\mathcal{G}))
$$

converges to $y^{\sharp}$ in $Y^{\sharp}$. Note that the filter

$$
i^{-1}\left(\iota_{Y}^{-1}(\mathcal{H})\right)
$$

is a Cauchy filter on $X$ so that $\iota_{X}\left(i^{-1}\left(\iota_{Y}^{-1}(\mathcal{H})\right)\right)$ must converge in $X^{\sharp}$ to some $x^{\sharp}$. But $\iota_{X}\left(i^{-1}\left(\iota_{Y}^{-1}(\mathcal{H})\right)\right) \subseteq \iota_{X}(\mathcal{F})$ and $\iota_{X}\left(i^{-1}\left(\iota_{Y}^{-1}(\mathcal{H})\right)\right) \subseteq \iota_{X}(\mathcal{G})$ so that $\iota_{X}(\mathcal{F})$ and $\iota_{X}(\mathcal{G})$ must converge to $x^{\sharp}$ as well. Since $X^{\sharp}$ is Hausdorff it follows by (5) that $x_{0}^{\sharp}=x_{1}^{\sharp}=x^{\sharp}$. Therefore $i^{\sharp}$ is injective.

It remains to verify that $i^{\sharp-1}$ is uniformly continuous on $i^{\sharp}\left(X^{\sharp}\right)$. This follows by similar arguments as those employed above.

Theorem 2.2. Let $X$ and $Y$ be uniform convergence spaces, and $F: X \rightarrow Y a$ uniformly continuous embedding. Then there exists a uniformly continuous embed$\operatorname{ding} F^{\sharp}: X^{\sharp} \rightarrow Y^{\sharp}$, where $X^{\sharp}$ and $Y^{\sharp}$ are the completions of $X$ and $Y$ respectively, which extends $F$. 
Proof. Since $X$ is uniformly homeomorphic to the subspace $F(X)$ of $Y$, it follows that $X^{\sharp}$ is uniformly homeomorphic to $F(X)^{\sharp}$ which is a subspace of $Y^{\sharp}$ by Proposition 2.1. Hence we obtain the following two commutative diagrams.
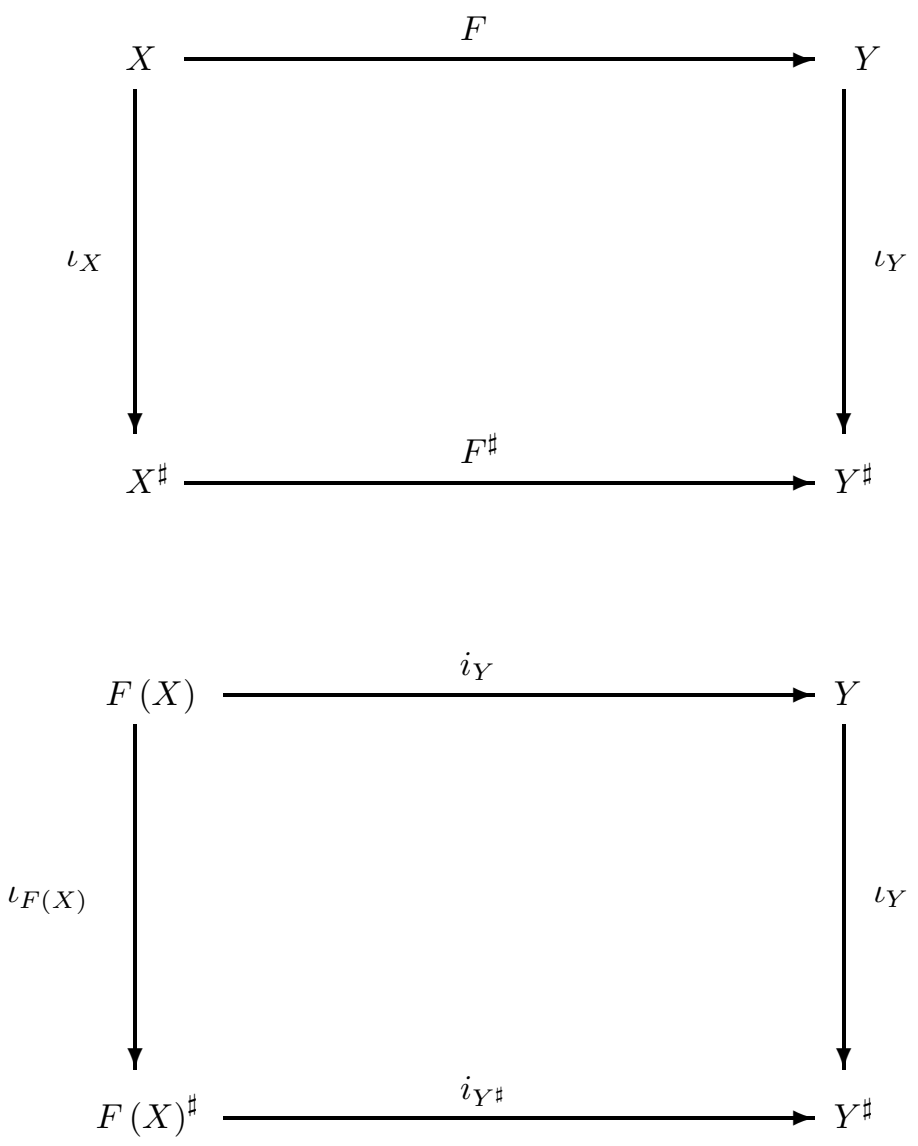

Here $i_{Y}$ and $i_{Y \sharp}$ denote the inclusion mappings on $F(X)$ and $F(X)^{\sharp}$, respectively. It now follows that $F^{\sharp}: X^{\sharp} \rightarrow Y^{\sharp}$ is an embedding, which completes the proof.

\section{Completion of Initial Uniform Convergence Spaces}

We now proceed to characterize the completion of a uniform convergence space $X$ which carries the initial uniform convergence structure $\mathcal{J}_{X}$ with respect to a family of mappings

$$
\left(\psi_{i}: X \rightarrow X_{i}\right)_{i \in I}
$$

where each of the sets $X_{i}$ is equipped with a Hausdorff uniform convergence structure $\mathcal{J}_{i}$. In this regard, we obtain a realization of the completion $X^{\sharp}$ of $X$ as a subspace of the completion of $\prod_{i \in I} X_{i}$, which carries the product uniform convergence structure [4, Section 2.2]. First, however, we characterize the completion of $\prod_{i \in I} X_{i}$. 
Theorem 3.1. Let $\left(X_{i}\right)_{i \in I}$ be a family of uniform convergence spaces and let $X$ denote their Cartesian product equipped with the product uniform convergence structure. Then the completion $X^{\sharp}$ of $X$ is the product of the completions $X_{i}^{\sharp}$ of the $X_{i}$.

Proof. First note that $\prod_{i \in I} X_{i}^{\sharp}$ is complete by 4, Proposition 2.3.3 (iii)]. For every $i$, let $\varphi_{i}: X_{i} \rightarrow X_{i}^{\sharp}$ be the uniformly continuous embedding associated with the completion $X_{i}^{\sharp}$ of $X_{i}$. Define the mapping $\varphi: X \rightarrow \prod X_{i}^{\sharp}$ through

$$
\varphi: x=\left(x_{i}\right) \mapsto\left(\varphi_{i}\left(x_{i}\right)\right)
$$

Also, for each $i$, let $\pi_{i}: X \rightarrow X_{i}$ be the projection.

Since each $\varphi_{i}$ is injective, so is $\varphi$. Moreover, we have

$$
\begin{aligned}
\mathcal{U} \in \mathcal{J}_{P} & \Rightarrow\left(\pi_{i} \times \pi_{i}\right)(\mathcal{U}) \in \mathcal{J}_{i} \\
& \Rightarrow\left(\varphi_{i} \times \varphi_{i}\right)\left(\left(\pi_{i} \times \pi_{i}\right)(\mathcal{U})\right) \in \mathcal{J}_{i}^{\sharp} \\
& \Rightarrow \prod_{i \in I}\left(\varphi_{i} \times \varphi_{i}\right)\left(\left(\pi_{i} \times \pi_{i}\right)(\mathcal{U})\right) \in \mathcal{J}_{P}^{\sharp} \\
& \Rightarrow(\varphi \times \varphi)(\mathcal{U}) \in \mathcal{J}_{P}^{\sharp}
\end{aligned}
$$

Hence $\varphi$ is uniformly continuous. Similarly, if the filter $\mathcal{V}$ on $\varphi(X) \times \varphi(X)$ belongs to the subspace uniform convergence structure, then

$$
\begin{aligned}
\left(\pi_{i} \times \pi_{i}\right)(\mathcal{V}) \in \mathcal{J}_{i}^{\sharp} & \Rightarrow\left(\varphi_{i}^{-1} \times \varphi_{i}^{-1}\right)\left(\left(\pi_{i} \times \pi_{i}\right)(\mathcal{V})\right) \in \mathcal{J}_{i} \\
& \Rightarrow \prod_{i \in I}\left(\varphi_{i}^{-1} \times \varphi_{i}^{-1}\right)\left(\left(\pi_{i} \times \pi_{i}\right)(\mathcal{V})\right) \in \mathcal{J}_{P} \\
& \Rightarrow\left(\varphi^{-1} \times \varphi^{-1}\right)(\mathcal{V}) \in \mathcal{J}_{P}
\end{aligned}
$$

so that $\varphi^{-1}$ is uniformly continuous. Hence $\varphi$ is a uniformly continuous embedding. That $\varphi(X)$ is dense in $\prod_{i \in I} X_{i}^{\sharp}$ follows by the denseness of $\varphi_{i}\left(X_{i}\right)$ in $X_{i}^{\sharp}$, for each $i \in I$. The extension property of uniformly continuous mappings into a complete uniform convergence space follows in the standard way.

The main result of this section is now the following.

Theorem 3.2. For each $i \in I$, let $X_{i}$ be a Hausdorff uniform convergence space, with uniform convergence structure $\mathcal{J}_{X_{i}}$. Let the uniform convergence space $X$ carry the initial uniform convergence structure $\mathcal{J}_{X}$ with respect to the family of mappings

$$
\left(\psi_{i}: X \mapsto X_{i}\right)_{i \in I}
$$

Assume that $\left(\psi_{i}\right)_{i \in I}$ separates the points of $X$. Then there exists a uniformly continuous embedding

$$
\Psi^{\sharp}: X^{\sharp} \rightarrow \prod_{i \in I} X_{i}^{\sharp}
$$


such that, for each $i \in I$, the diagram commutes:

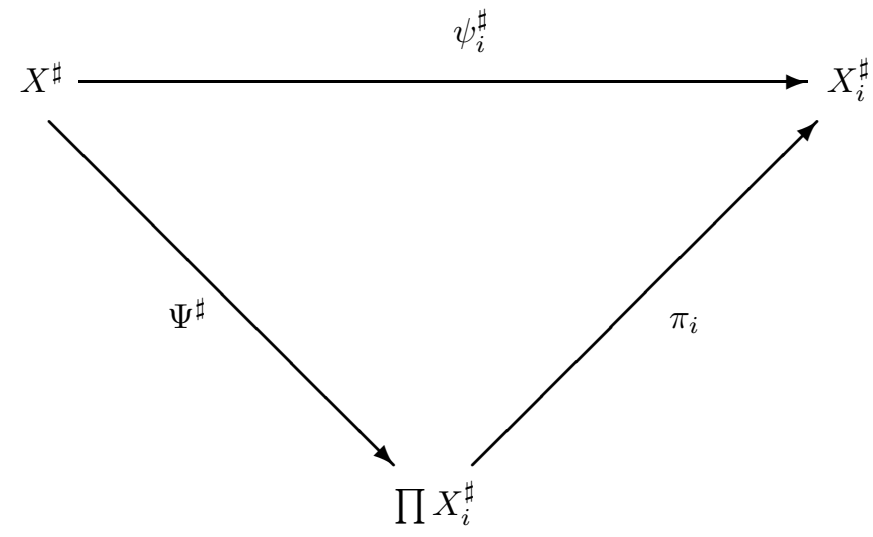

Proof. Define the mapping $\Psi$ as

$$
\Psi: X \ni x \mapsto\left(\psi_{i}(x)\right)_{i \in I} \in \prod_{i \in I} X_{i}
$$

Clearly, the diagram

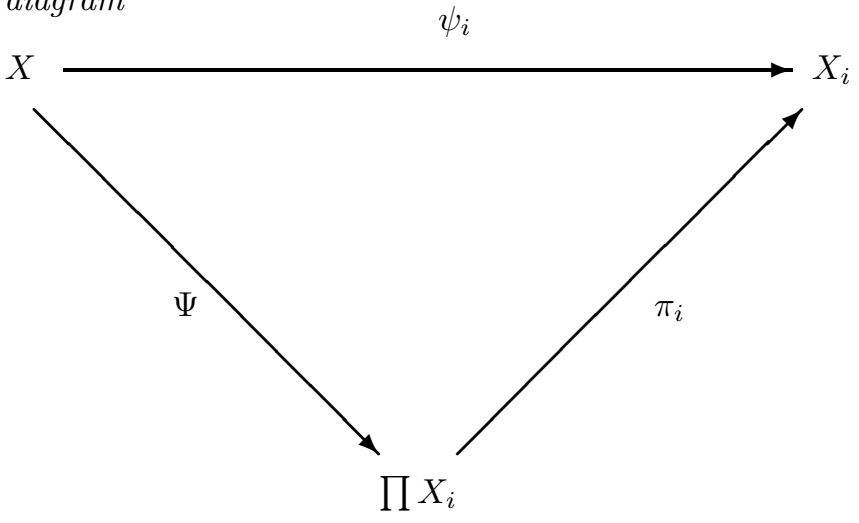

commutes for every $i \in I$. Suppose that $\mathcal{U} \in \mathcal{J}_{X}$. Then

$$
\begin{aligned}
\forall \quad & i \in I: \\
& \left(\psi_{i} \times \psi_{i}\right)(\mathcal{U}) \in \mathcal{J}_{X_{i}}:
\end{aligned}
$$

so that

$$
\begin{aligned}
& \forall \quad i \in I: \\
& \quad\left(\pi_{i} \times \pi_{i}\right)(\Psi \times \Psi)(\mathcal{U}) \in \mathcal{J}_{X_{i}}:
\end{aligned}
$$

Therefore $(\Psi \times \Psi)(\mathcal{U}) \in \mathcal{J}_{\Pi}$, which is the product uniform convergence structure, so that $\Psi$ is uniformly continuous. Since $\left(\psi_{i}\right)_{i \in I}$ separates the points of $X, \Psi$ is injective. Let $\mathcal{V} \in \mathcal{J}_{P}$ be a filter on $\prod_{i \in I} X_{i} \times \prod_{i \in I} X_{i}$ with a trace on $\Psi(X) \times \Psi(X)$. 
Then

$$
\begin{aligned}
& \forall \quad i \in I: \\
& \quad \text { a) }\left(\pi_{i} \times \pi_{i}\right)(\mathcal{V}) \in \mathcal{J}_{X_{i}} \\
& \text { b) } W \in\left(\pi_{i} \times \pi_{i}\right)(\mathcal{V}) \Rightarrow W \cap\left(\psi_{i}(X) \times \psi_{i}(X)\right) \neq \emptyset
\end{aligned}
$$

so that

$$
\begin{aligned}
& \forall \quad i \in I: \\
& \quad\left(\psi_{i} \times \psi_{i}\right)\left(\left(\Psi^{-1} \times \Psi^{-1}\right)(\mathcal{V})\right) \supseteq\left(\pi_{i} \times \pi_{i}\right)(\mathcal{V})
\end{aligned}
$$

Form the definition of an initial uniform convergence structure, and in particular the product uniform convergence structure, it follows that $\left(\Psi^{-1} \times \Psi^{-1}\right)(\mathcal{V}) \in \mathcal{J}_{X}$. Hence $\Psi$ is a uniformly continuous embedding. The result now follows form Proposition 2.1 and Theorem 3.1.

The following is now a straight forward consequence of Theorem 3.2 .

Corollary 3.3. Let $X$ carry the initial uniform convergence structure with respect to the family

$$
\left(\psi_{i}: X \rightarrow X_{i}\right)_{i \in I}
$$

of mappings, where each $X_{i}$ is a Hausdorff uniform convergence structure. Then the the completion $X^{\sharp}$ carries the initial uniform convergence structure with respect to the family of mappings

$$
\left(\psi_{i}^{\sharp}: X^{\sharp} \rightarrow X_{i}^{\sharp}\right)_{i \in I}
$$

\section{An Application to Nonlinear PDEs}

As an application of the results obtained in Sections 2 and 3, we prove an existence result for generalized solutions to arbitrary $\mathcal{C}^{\infty}$-smooth PDEs. In this regard, consider a nonlinear PDE

$$
T(x, D) U(x)=f(x), x \in \Omega
$$

of order $m$, where $\Omega \subseteq \mathbf{R}^{n}$ is some nonempty open subset of $\mathbf{R}^{n}$. The right hand term $f$ is assumed to be a $\mathcal{C}^{\infty}$-smooth function on $\Omega$, while the PDE operator $T(x, D)$ is supposed to be defined through a $\mathcal{C}^{\infty}$-smooth function

$$
F: \Omega \times \mathbf{R}^{K} \rightarrow \mathbf{R}
$$

by

$$
\begin{aligned}
\forall & u \in \mathcal{C}^{\infty}(\Omega): \\
\forall & x \in \Omega: \\
& T(x, D) u(x)=F\left(x, u(x), \ldots, D^{\alpha} u(x), \ldots\right),|\alpha| \leq m
\end{aligned}
$$

We also make the following technical assumption:

$$
\begin{aligned}
\forall \quad & x \in \Omega: \\
& f(x) \in \operatorname{int}\left\{F(x, \xi): \xi \in \mathbf{R}^{K}\right\}
\end{aligned}
$$

Note that (10) is merely a a necessary condition for the existence of a classical solution to (8) on a neighborhood of $x \in \Omega$. 
We construct generalized solutions to (8) which may be assimilated with functions which are $\mathcal{C}^{\infty}$-smooth everywhere on $\Omega$, except on a closed nowhere dense set. In this regard, consider the space, see [11] or [12]

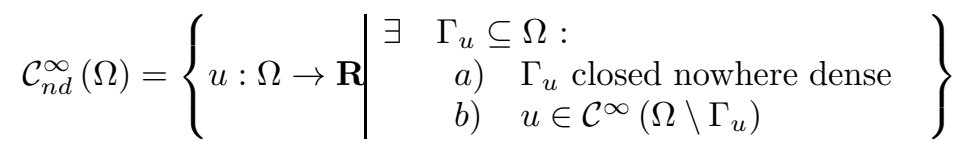

and the equivalence relation

$$
\begin{aligned}
& \forall u, v \in \mathcal{C}^{\infty}(\Omega): \\
& u \sim v \Leftrightarrow\left(\begin{array}{rrl}
\exists & \Gamma_{u v} \subseteq \Omega: \\
& a) & \Gamma_{u v} \text { closed nowhere dense } \\
& b) & u, v \in \mathcal{C}^{\infty}\left(\Omega \backslash \Gamma_{u v}\right) \\
c) & x \in \Omega \backslash \Gamma \Rightarrow u(x)=v(x)
\end{array}\right)
\end{aligned}
$$

The quotient space $\mathcal{C}_{n d}^{\infty}(\Omega) / \sim$ is denoted $\mathcal{M}^{\infty}(\Omega)$. A partial order [1] on $\mathcal{M}^{\infty}(\Omega)$ may be defined through

$$
\begin{aligned}
& \forall \quad U, V \in \mathcal{M}^{\infty}(\Omega): \\
& \quad U \leq V \Leftrightarrow\left(\begin{array}{cc}
\exists & u \in U, v \in V: \\
\exists & \Gamma_{u v} \subset \Omega \text { closed nowhere dense : } \\
& a) \quad u, v \in \mathcal{C}^{\infty}\left(\Omega \backslash \Gamma_{u v}\right) \\
& b) \quad x \in \Omega \backslash \Gamma_{u v} \Rightarrow u(x) \leq v(x)
\end{array}\right)
\end{aligned}
$$

It is easy to see that $\mathcal{M}^{\infty}(\Omega)$, equipped with the order (13) is in fact a vector lattice [9], and hence it is fully distributive.

In view of (8) and (11), it is clear that we have the mapping

$$
T(x, D): \mathcal{C}_{n d}^{\infty}(\Omega) \rightarrow \mathcal{C}_{n d}^{\infty}(\Omega)
$$

Moreover,

$$
\begin{aligned}
& \forall \quad u, v \in \mathcal{C}_{n d}^{\infty}(\Omega): \\
& \quad u \sim v \Rightarrow T(x, D) u \sim T(x, D) v
\end{aligned}
$$

so that one may associate with the PDE operator $T(x, D)$ a mapping

$$
T: \mathcal{M}^{\infty}(\Omega) \rightarrow \mathcal{M}^{\infty}(\Omega)
$$

in a canonical way. On the space $\mathcal{M}^{\infty}(\Omega)$, define the equivalence relation

$$
\begin{aligned}
& \forall \quad U, V \in \mathcal{M}^{\infty}(\Omega): \\
& U \sim_{T} V \Leftrightarrow T U=T V
\end{aligned}
$$

and denote the quotient space $\mathcal{M}^{\infty}(\Omega) / \sim_{T}$ by $\mathcal{M}_{T}^{\infty}(\Omega)$. We may associate with the mappint $T$ an injective mapping

$$
\widehat{T}: \mathcal{M}_{T}^{\infty}(\Omega) \rightarrow \mathcal{M}^{\infty}(\Omega)
$$

so that the diagram 


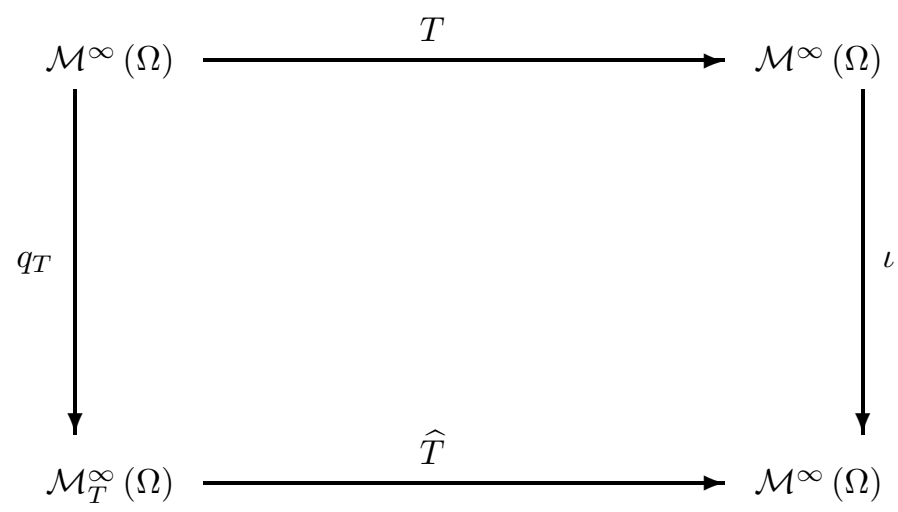

commutes, with $q_{T}$ the quotient mapping associated with $\sim_{T}$, and $\iota$ the identity.

We now introduce a uniform convergence structure on $\mathcal{M}^{\infty}(\Omega)$. This is achieved by first defining a convergence structure on $\mathcal{M}^{\infty}(\Omega)$.

Definition 4.1. For every $U \in \mathcal{M}^{\infty}(\Omega)$, define the family $\lambda_{o}(U)$ on $\mathcal{M}^{\infty}(\Omega)$ as follows:

$\forall \mathcal{F}$ a filter on $\mathcal{M}^{\infty}(\Omega)$ :

$$
\mathcal{F} \in \lambda_{o}(U) \Leftrightarrow\left(\begin{array}{ll}
\forall & n \in \boldsymbol{N}: \\
\exists & {\left[L_{n}, U_{n}\right] \subset \mathcal{M}^{\infty}(\Omega):} \\
a) & n \in \boldsymbol{N} \Rightarrow\left[L_{n+1}, U_{n+1}\right] \subseteq\left[L_{n}, U_{n}\right] \\
\text { b) } & \sup \left\{L_{n}: n \in \boldsymbol{N}\right\}=U=\inf \left\{U_{n}: n \in \boldsymbol{N}\right\} \\
\text { c) } & {\left[\left\{\left[L_{n}, U_{n}\right]: n \in \boldsymbol{N}\right\}\right] \subseteq \mathcal{F}}
\end{array}\right)
$$

That $\lambda_{o}$ is indeed a convergence structure follows by [2, Theorem 17]. Moreover, $\lambda_{o}$ is Hausdorff. It now follows that the associated uniform convergence structure $\mathcal{J}_{\lambda_{o}}$, 4, Proposition 2.1.7], namely

$$
\forall \mathcal{U} \text { a filter on } \mathcal{M}^{\infty}(\Omega) \times \mathcal{M}^{\infty}(\Omega) \text { : }
$$

$$
\mathcal{U} \in \mathcal{J}_{\lambda_{o}} \Leftrightarrow\left(\begin{array}{cc}
\exists & \mathcal{F}_{1}, \ldots, \mathcal{F}_{k} \text { filters on } \mathcal{M}^{\infty}(\Omega): \\
\exists & U_{1}, \ldots, U_{k} \in \mathcal{M}^{\infty}(\Omega): \\
& a) \quad \mathcal{F}_{i} \in \lambda_{o}\left(U_{i}\right), i=1, \ldots, k \\
\text { b) } & \mathcal{U} \supseteq\left(\mathcal{F}_{1} \times \mathcal{F}_{1}\right) \cap \ldots \cap\left(\mathcal{F}_{k} \times \mathcal{F}_{k}\right)
\end{array}\right)
$$

is uniformly Hausdorff and complete. The space $\mathcal{M}_{T}^{\infty}(\Omega)$ will carry the initial uniform convergence structure with respect to the mapping

$$
\widehat{T}: \mathcal{M}^{\infty}(\Omega) \rightarrow \mathcal{M}_{T}^{\infty}(\Omega)
$$

The following is now immediate:

Proposition 4.2. The mapping $\widehat{T}$ is an embedding of the uniform convergence space $\mathcal{M}_{T}^{\infty}(\Omega)$ into the uniform convergence space $\mathcal{M}^{\infty}(\Omega)$.

As in the rest of the paper, we denote by $\mathcal{M}^{\infty}(\Omega)^{\sharp}$ and $\mathcal{M}_{T}^{\infty}(\Omega)^{\sharp}$ the uniform convergence space completions of $\mathcal{M}^{\infty}(\Omega)$ and $\mathcal{M}_{T}^{\infty}(\Omega)$, respectively. The extension of the uniformly continuous embedding

$$
\widehat{T}: \mathcal{M}_{T}^{\infty}(\Omega) \rightarrow \mathcal{M}^{\infty}(\Omega)
$$


is denoted $\widehat{T}^{\sharp}$. The generalized equation, corresponding to (8), now takes the form

$$
\widehat{T}^{\sharp} U^{\sharp}=f
$$

A solution $U^{\sharp}$ to (19) is interpreted as generalized solution to (8).

We recall the following basic approximation result 11 .

Theorem 4.3. Consider a PDE of the form (8) through (9) that also satisfies (10). For every $\epsilon>0$ there exists a closed nowhere dense set $\Gamma_{\epsilon} \subset \Omega$ with zero Lebesgue measure, and a function $u_{\epsilon} \in \mathcal{C}^{\infty}\left(\Omega \backslash \Gamma_{\epsilon}\right)$ such that

$$
f(x)-\epsilon \leq T(x, D) u_{\epsilon}(x) \leq f(x), x \in \Omega \backslash \Gamma_{\epsilon}
$$

The main result of this section now follows.

Theorem 4.4. Consider a nonlinear PDE of the form (8) through (9). For every $f \in \mathcal{C}^{\infty}(\Omega)$ that satisfies (10), there exists a unique $U^{\sharp} \in \mathcal{M}_{T}^{\infty}(\Omega)^{\sharp}$ such that

$$
\widehat{T}^{\sharp} U^{\sharp}=f
$$

Proof. First let us show existence. For every $n \in N$, Theorem 4.3 yields a closed nowhere dense set $\Gamma_{n} \subset \Omega$ and a function $u_{n} \in \mathcal{C}^{\infty}\left(\Omega \backslash \Gamma_{n}\right)$ that satisfies

$$
x \in \Omega \backslash \Gamma_{n} \Rightarrow f(x)-\frac{1}{n} \leq T(x, D) u_{n}(x) \leq f(x)
$$

Since $\Gamma_{n}$ is closed nowhere dense we associate $u_{n}$ with the equivalence class $U_{n} \in$ $\mathcal{M}^{\infty}(\Omega)$ in a unique way.

Denote by $V_{n}$ the equivalence class generated by $U_{n}$ under the equivalence relation $\sim_{T}$. Clearly, the sequence $\left(\widehat{T} V_{n}\right)$ is increasing in $\mathcal{M}^{\infty}(\Omega)$ and has upper bound $f$. In fact, $f$ is the least upper of the sequence $\left(\widehat{T} V_{n}\right)$. Hence $\left(\widehat{T} V_{n}\right)$ converges to $f$ in $\mathcal{M}^{\infty}(\Omega)$. It now follows that $\left(V_{n}\right)$ is a Cauchy sequence in $\mathcal{M}_{T}^{\infty}(\Omega)$ so that there exists $U^{\sharp} \in \mathcal{M}_{T}^{\infty}(\Omega)$ that satisfies (19).

Since the mapping $\widehat{T}: \mathcal{M}_{T}^{\infty}(\Omega) \rightarrow \mathcal{M}^{\infty}(\Omega)$ is a uniformly continuous embedding, the uniqueness of the solution $U^{\sharp}$ found above now follows by Theorem 2.2.

Note that the uniqueness of the generalized solution $U^{\sharp}$ to (19) should not be misinterpreted as implying that any, possibly classical, solutions are disregarded. In fact, quite the contrary. Recall that the completion of a uniform convergence space $X$ may be obtained constructively. In particular, it consists of all equivalence classes of Cauchy filters on $X$ so that the members of an equivalence class $[\mathcal{F}]$ all convergence to the same element of the completion $X^{\sharp}$. In view of this, the unique generalized solution is in fact the totality of all approximate solutions in $\mathcal{M}^{\infty}(\Omega)$.

Notice also that the mapping

$$
\widehat{T}^{\sharp}: \mathcal{M}_{T}^{\infty}(\Omega)^{\sharp} \rightarrow \mathcal{M}^{\infty}(\Omega)
$$

is an embedding. Hence, as a bonus, we also have a blanket regularity in the sense that every element $U^{\sharp}$ of $\mathcal{M}_{T}^{\infty}(\Omega)^{\sharp}$ may be assimilated with elements of $\mathcal{M}^{\infty}(\Omega)$.

\section{Conclusion}

In a rather straightforward way, we have established a characterization of the completion of an initial uniform convergence space. The ease with which this result follows is in fact typical of many of the constructions in the theory of convergence 
space, which contains that of the usual topology as a special case. This is in contradistinction to topology, with the associated elaborate constructions.

As an application of these results, we obtain the existence of generalized solutions to arbitrary, nonlinear $\mathcal{C}^{\infty}$-smooth PDEs. In addition, a blanket regularity result is obtained, in the sense that every generalized solution may be assimilated with functions which are $\mathcal{C}^{\infty}$-smooth everywhere except on a closed nowhere dense set.

\section{ACKNOWLEDGEMENT}

The author would like to thank Professors E. E. Rosinger and R. Anguelov for their comments and suggestions.

\section{REFERENCES}

[1] R. Anguelov and E. E. Rosinger, Solving large classes of nonlinear systems of PDE's (Computers and Mathematics with Applications 53 (2007) 491-507).

[2] R. Anguelov and J. H. van der Walt, Order convergence structure on $\mathcal{C}(X)$ (Quaestiones Mathematicae 28 No. 4 (2005), 425-457).

[3] R. Beattie, Continuous convergence and functional analysis (Topology and its applications 70 (1996), 101-111).

[4] R. Beattie and H. P. Butzmann, Convergence structures and applications to functional analysis (Kluwer Academic Plublishers, Dordrecht, Boston, London, 2002).

[5] W. Gähler, Grundstrukturen der anlysis I (Birkhäuser Verlag, Basel, 1977).

[6] W. Gähler W, Grundstrukturen der anlysis II (Birkhäuser Verlag, Basel, 1978).

[7] S. Gähler, W. Gähler and G. Kneis, Completion of pseudo-topological vector spaces (Mathematische Nachrichten bf 75 (1976), 185-206).

[8] J. Kelley, General topology (Van Nostrand, Princeton, 1955).

[9] W. A. Luxemburg and A. C. Zaanen, Riesz Spaces I (North-Holland, Amsterdam, London, 1971).

[10] R. A. McCoy and I. Ntantu, Topological properties of spaces of continuous functions (Lecture notes in mathematics 1315, Springer-Verlag 1988).

[11] M. Oberguggenberger and E. E. Rosinger, Solution of continuous nonlinear PDEs through order completion (North-Holland, Amsterdam, London, New York, Tokyo, 1994).

[12] Rosinger, E. E.: Nonlinear partial differential equations, an algebraic view of generalized solutions, North Holland Mathematics Studies, vol. 164, 1990.

[13] E. E. Rosinger and J. H. van der Walt, Beyond topology (to appear).

[14] J. H. van der Walt, The order completion method for systems of nonlinear PDEs: Pseudotopological perspectives (Technical Report UPWT 2007/07, University of Pretoria).

[15] J H. van der Walt, Generalized functions and nonlinear PDEs (To Appear).

Department of Mathematics and Applied Mathematics, University of Pretoria, Pretoria 0002, South Africa 